\title{
Superb Microvascular Imaging of Retained Placenta with Placenta Accreta Spectrum
}

\author{
Toshiyuki Hata ${ }^{1}$, Uiko Hanaoka ${ }^{2}$, Ayumi Mori ${ }^{3}$, Kenta Yamamoto ${ }^{4}$, Chiaki Tenkumo ${ }^{5}$, Nobuhiro Mori ${ }^{6}$, Kenji Kanenishi ${ }^{7}$,
} Hirokazu Tanaka ${ }^{8}$

\begin{abstract}
We present our first experience of using superb microvascular imaging (SMI) with an 18-MHz linear probe to diagnose placenta accreta spectrum (PAS) in the retained placenta after vaginal delivery. In the first case, irregular minimal invasion of the retained placenta into the anterior myometrium and a thin uterine wall were clearly noted. In the second case, SMI clearly demonstrated the loss of myometrium anterior to fundal lesions and abnormally dilated, torturous, stem villous vessels invading until the uterine serosa. SMI with the use of an 18-MHz linear probe may be a novel diagnostic tool for the diagnosis of PAS in the retained placenta after delivery.

Keywords: 18-MHz linear probe, High-resolution ultrasound, Placenta accreta spectrum, Retained placenta, Superb microvascular imaging. Donald School Journal of Ultrasound in Obstetrics and Gynecology (2019): 10.5005/jp-journals-10009-1600
\end{abstract}

\section{INTRODUCTION}

A placenta accreta spectrum (PAS) is one of the most serious and potentially life-threatening conditions for both a pregnant woman and fetus. ${ }^{1}$ The precise prenatal diagnosis of PAS improves the prognosis of the patient and reduces maternal morbidity. ${ }^{2}$ The European Working Group on PAS has proposed standardized ultrasound descriptors of this spectrum. ${ }^{3}$ However, the heterogeneity in terminology and differences in study design limit the assessment of the accuracy of ultrasound imaging in the screening and diagnosis of PAS. ${ }^{4}$ Therefore, standardized protocols for screening, diagnosis, and management of PAS need to be developed. ${ }^{5}$

Superb microvascular imaging (SMI) is the latest Doppler technology, and it can detect lower blood flow velocity without motion artifacts. ${ }^{6,7}$ Moreover, SMI provides high-resolution ultrasound images at high frame rates. There have been only three SMI studies on normal and abnormal placental vasculatures. ${ }^{8-10}$ Moreover, there has been only one report to diagnose PAS with SMI using an $18-24 \mathrm{MHz}$ probe during pregnancy. ${ }^{11}$ Here, we present our first experience of using conventional SMI and high-resolution SMI with an $18-\mathrm{MHz}$ linear probe to diagnose PAS in the retained placenta after delivery.

\section{Case Description}

\section{Case 1}

A 37-year-old Japanese woman was referred to our university hospital because of retained placenta and excessive postpartum bleeding after delivery. Two-dimensional (2D) sonography and conventional SMI (Aplio i800, Canon Medical Systems, Tokyo, Japan) showed suspicious signs of PAS at the anterior uterine wall (Fig. 1). MRI could not clearly demonstrate the PAS. High-resolution SMI with an 18-MHz linear probe clearly depicted irregular minimal invasion of the retained placenta into the anterior myometrium, and a thin uterine wall was noted (Fig. 2A). High-resolution SMI also revealed disruption of decidual vessels (DV) at this pathological lesion, and abnormal lacunar flow was also noted (Fig. 2B). Ultrasound-guided removal under general anesthesia was performed, and removal of the placenta was successful. Histopathological diagnosis of the removed placenta was placenta accreta (the villi attached directly to the myometrial tissue without intermediate decidua).

\footnotetext{
${ }^{1-8}$ Department of Perinatology and Gynecology, Kagawa University Graduate School of Medicine, Miki, Kagawa, Japan

Corresponding Author: Toshiyuki Hata, Department of Perinatology and Gynecology, Kagawa University Graduate School of Medicine, Miki, Kagawa, Japan, Phone: +81-87-891-2174, e-mail: toshi28@med. kagawa-u.ac.jp

How to cite this article: Hata T, Hanaoka U, et al. Superb Microvascular Imaging of Retained Placenta with Placenta Accreta Spectrum. Donald School J Ultrasound Obstet Gynecol 2019;13(3):85-87.

Source of support: Nil

Conflict of interest: None
}

\section{Case 2}

A 40-year-old Japanese woman spontaneously aborted in our university hospital at 18 weeks and 6 days of gestation. She had undergone enucleation of uterine adenomyosis at another hospital 20 months previously. After she delivered, the placenta remained in situ. Two-dimensional sonography showed broad anechoic lesions in the uterus, and conventional SMI revealed abundant blood flow in the placental bed adjacent to the uterine wall (Fig. 3). MRI suggested placenta increta (placental invasion to the anterior myometrium and thinner uterine wall at the anterior fundal lesion). High-resolution SMI with an $18-\mathrm{MHz}$ probe clearly demonstrated the loss of myometrium anterior to fundal lesions and abnormally dilated, torturous, stem villous vessels invading until the uterine serosa (snow storm-like appearance) (Fig. 4). There was no excessive vaginal bleeding. The placenta was left in situ, and the patient was discharged without any adverse events. The patient is now scheduled for monthly scans until the placenta has been completely reabsorbed.

\section{Discussion}

Retained placenta after vaginal delivery is a rare complication associated with increasing maternal morbidity and mortality. ${ }^{12}$ Common causes of retained placenta are PAS. However, there is no consensus concerning acceptable interobserver variability of ultrasound signs. ${ }^{13}$ One possible explanation for the inconsistent 


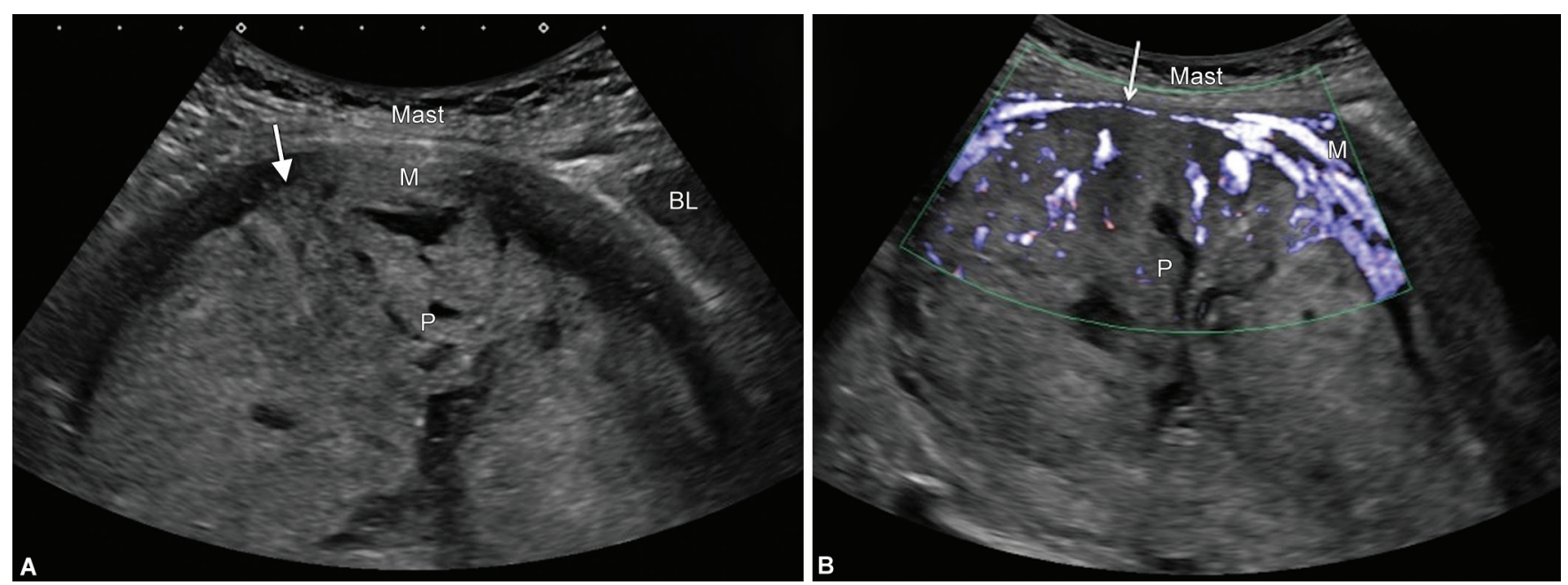

Figs $1 \mathrm{~A}$ and B: Retained placenta with placenta accreta. Two-dimensional sonography (A) and conventional SMI (B) show suspicious signs of PAS at the anterior uterine wall (arrow). M, myometrium; MASF, maternal abdominal subcutaneous fat; $P$, placenta


Figs $2 \mathrm{~A}$ and B: Retained placenta with placenta accreta. High-resolution ultrasound with an 18-MHz linear probe clearly demonstrates irregular minimal invasion of the retained placenta into the anterior myometrium (arrows), and a thin uterine wall is noted (A). Superb microvascular imaging with an 18- $\mathrm{MHz}$ linear probe reveals a minimally invasive placenta in the anterior myometrium (arrows) and the disruption of DV (B). Abnormal lacunar flow is also noted. M, myometrium; MAM, maternal abdominal muscle; MASF, maternal abdominal subcutaneous fat; P, placenta

diagnostic criteria is the low resolution of conventional sonographic probes. Moreover, many of the ultrasound signs for the diagnosis of PAS are indirect findings. ${ }^{3}$ In the present study, we used highresolution SMI with an 18-MHz transabdominal probe and obtained the direct findings of irregular minimal invasion of the retained placenta into the anterior myometrium in the first case. In the second case, the loss of myometrium anterior to fundal lesions was clearly visualized. Moreover, this technique clearly showed the disruption of DV and abnormal lacunar flow in the placenta of the first case, and abnormally dilated, torturous, stem villous vessels (snow storm-like appearance) invading until the uterine serosa were evident in the second case. In the previous investigation on the antenatal diagnosis of PAS using high-resolution SMI with an 18-24 MHz probe, unique characteristic of PAS was dilated stem villous vessels at the lesion of placenta increta. ${ }^{11}$ These results suggest that high-resolution SMI using an $18-\mathrm{MHz}$ probe may also
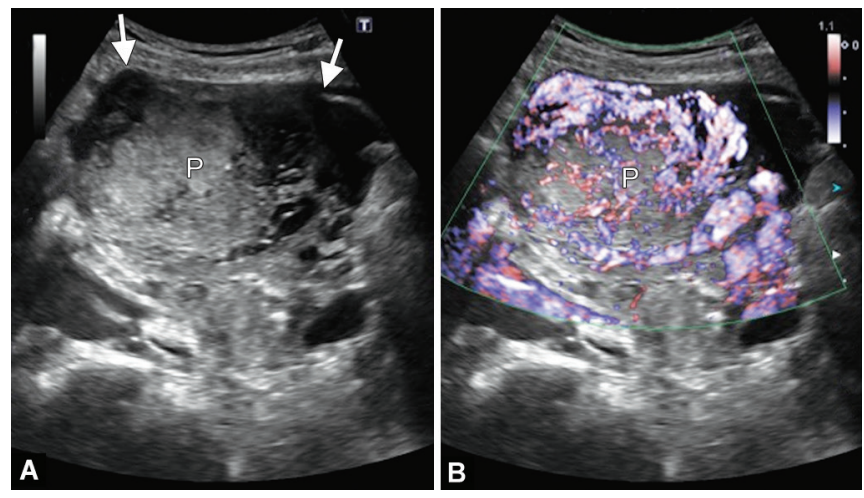

Figs $3 \mathrm{~A}$ and B: Retained placenta with placenta increta/percreta. Twodimensional sonography shows broad anechoic lesions in the uterus (arrows) (A), and conventional SMI reveals abundant blood flow in the placental bed adjacent to the uterine wall (B). P, placenta 

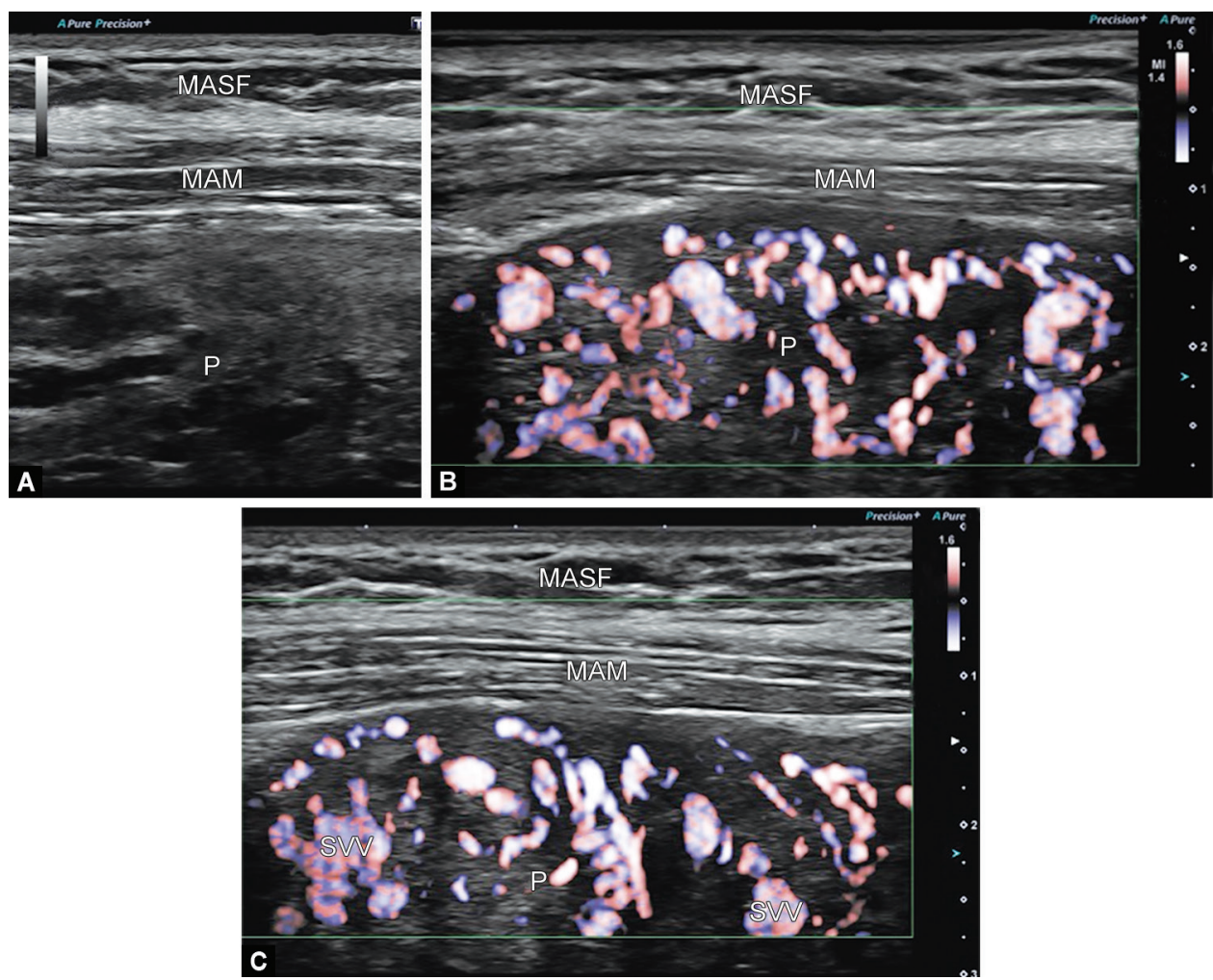

Figs 4A to C: Retained placenta with placenta increta/percreta. High-resolution ultrasound (A) and SMI (B and C) with an 18-MHz probe clearly demonstrate the loss of myometrium anterior to fundal lesions. Two-dimensional sonography depicts abnormally dilated, torturous vessels (A). Abnormally torturous, abundant blood flow (snow storm-like appearance) invading until the uterine serosa is evident (B). Abnormally dilated stem villous vessels (STV) are also evident (C). MAM, maternal abdominal muscle; MASF, maternal abdominal subcutaneous fat; $P$, placenta

be a novel diagnostic modality for the precise diagnosis of PAS in the retained placenta after delivery. Further studies involving a lager sample size are needed to clarify the usefulness of high-resolution $\mathrm{SMI}$ with an 18-MHz probe in the correct diagnosis of PAS during or after delivery.

\section{References}

1. Papanikolaou IG, Domail E, et al. Abnormal placentation: current evidence and review of the literature. Eur J Obstet Gyneco Reprod Biol 2018;228:98-105. DOI: 10.1016/j.ejogrb.2018. 06.004.

2. Buca $D$, Liberati $M$, et al. Influence of prenatal diagnosis of abnormally invasive placenta on maternal outcome: a systematic review and meta-analysis. Ultrasound Obstet Gynecol 2018;52(3):304-309. DOI: 10.1002/uog.19070.

3. Collins SL, Ashcroft A, et al. Proposal for standardized ultrasound descriptors of abnormally invasive placenta (AIP). Ultrasound Obstet Gynecol 2016;47:271-275. DOI: 10.1002/uog.14952.

4. Jauniaux E, Collins SL, et al. Accreta placentation: a systematic review of prenatal ultrasound imaging and grading of villous invasiveness. Am J Obstet Gynecol 2016;215:712-721. DOI: 10.1016/ j.ajog.2016.07.044.

5. Jauniaux E, Collins SL, et al. Placenta accreta spectrum: pathophysiology and evidence-based anatomy for prenatal ultrasound imaging. Am J Obstet Gynecol 2018;218:75-87. DOI: 10.1016/j.ajog. 2017.05.067.

6. Wu L, Yen $\mathrm{HH}$, et al. Spoke-wheel sign of focal nodular hyperplasia revealed by superb micro-vascular ultrasound imaging. QJM 2015;108:669-670. DOI: 10.1093/qjmed/hcv016.

7. Ma Y, Li G, et al. The diagnostic value of superb microvascular imaging (SMI) in detecting blood flow signals of breast lesions. Medicine (Baltimore) 2015;94(36):e1502. DOI: 10.1097/MD.0000000000001502.

8. Hasegawa J, Suzuki N. SMI for imaging of placental infarction. Placenta 2016;47:96-98. DOI: 10.1016/j.placenta.2016.08.092.

9. Hasegawa J, Yamada H, et al. Application of superb microvascular imaging (SMI) in obstetrics. J Matern Fetal Neonatal Med 2018;31: 261-263. DOI: 10.1080/14767058.2016.1278206.

10. Hata $T$, Kanenishi $K$, et al. Microvascular imaging of thick placenta with fetal growth restriction. Ultrasound Obstet Gynecol 2018;51:837-839. DOI: 10.1002/uog.18837.

11. Hasegawa J, Kurasaki A, et al. Sono-histological findings of the placenta accreta spectrum. Ultrasound Obstet Gynecol 2018. DOI: 10.1002/uog.20207.

12. Bjurstrom J, Collins $S$, et al. Failed manual removal of the placenta after vaginal delivery. Arch Gynecol Obstet 2018;297:323-332. DOI: 10.1007/s00404-017-4579-2.

13. Thurn $L$, Lindqvist $P G$, et al. Abnormally invasive placenta-prevalence, risk factors and antenatal suspicion: results from a large populationbased pregnancy cohort study in the Nordic countries. BJOG 2016;123:1348-1355. DOI: 10.1111/1471-0528.13547. 